\title{
Virtual Teams, Organizations, and Networks (VTONs)
}

\author{
Derrick L. Cogburn \\ American University \\ dcogburn@american.edu
}

\author{
J. Alberto Espinosa \\ American University \\ alberto@american.edu
}

\author{
Alecia M. Santuzzi \\ Northern Illinois University \\ maguilar@american.edu
}

\begin{abstract}
Teamwork in organizations today is frequently conducted by individuals working across multiple spatial and temporal boundaries in complex configurations and mediated by technology. These virtual teams are typically characterized by multi-team memberships, member turnover, and diverse, porous organizational boundaries. Contemporary virtual teams can rarely be studied as single units because they are often co-mingled into larger organizational networks with multiple teams, locations, and organizational overlap. Most business, government, and scientific projects and processes today have very prominent virtual dimensions. Virtual collaborators often: do not have the same first language; come from different national cultures; work in different time zones, are employed by different organizations; and enter collaborations with different expectations for group processes. These differences, among others, often lead to fault lines and also present unique opportunities for management and leadership of virtual teams, organizations, and networks. This HICSS-52 mini-track explores many of these questions, using a wide variety of research methods and approaches.
\end{abstract}

\section{Introduction}

Teamwork in organizations today frequently involves members working across multiple spatial and temporal boundaries in complex configurations comprised of multi-team memberships, member turnover, and multiple organizational boundaries, among other things. Contemporary virtual teams can rarely be studied as single units because they are often co-mingled into larger organizational networks with multiple teams, locations, and organizational overlap. Most business, government, and scientific projects and processes today have a very prominent virtual dimension. Virtual collaborators often do not have the same first language, come from different national cultures, work in different time zones, may be employed by different organizations, and enter collaborations with different expectations for group processes. These differences, among others, present unique opportunities for management and leadership.

Because of the multi-disciplinary nature of research on virtual teams, organizations, and networks (VTONs), we encouraged submissions that may inform practice and research in virtual collaboration through a variety of academic lenses. We also encouraged papers discussing methodological issues and innovation to address the complexity in the study of virtual teams, organizations, and networks. This mini-track includes papers that offer direct and indirect insights into the successful operation of virtual teams, organizations and networks, including research in the vein of computer supported collaborative work (CSCW), computer supported collaborative learning (CSCL), and social networks.

\section{Overview of VTONs Topics and Themes}

Because VTONs are permeate all aspects of organizational collaboration, there are a wide variety of topics and themes associated with this mini-track and present in most of the featured papers. These topics include themes like: spatial, temporal, cultural and organizational dimensions; separation and their effects on communication and collaboration; team coordination, outcomes and performance; multi-cultural issues; shared leadership and power distance; trust, deception, emotion, personality and social loafing; innovation, learning and training; and social and organizational networking, multi-team systems and team science.

Our call for papers this year yielded a variety of excellent papers that covered many of these topics. This mini-track includes the four best papers identified by peer review. Collectively, the selected papers present research and practical lessons about the effective use of technology to support collaboration. The papers also present experiences from the individual user and team level perspectives, both of which are essential to a comprehensive understanding of virtual collaborations.

In the following sections, we present a brief summary of the VTONs papers to be presented at HICSS-52, including our Best Paper nomination. 


\section{Paper 1: International Virtual Research Organization Network Evolution}

The first paper explores the evolution of International Virtual Research Organizations (IVROs). IVROs are organizations established to foster collaboration between international groups using cyberinfrastructure, which provides mechanisms for organizing, planning, and executing scientific research. This study traces the evolution of the organizational network of a large multi-disciplinary IVRO over the course of nine years. Results show significant deviations in participation during certain years that may indicate organizational turbulence.

\section{Paper 2: Coordination in Distributed, Self-Managing Work Teams}

The second paper tackles the important element of coordination, with a focus on distributed self-managing work teams. The study investigates team selfmanagement and team members' perceived coordination in the context of distributed teams that rely on electronic communication tools to interact. In addition, this study examines the moderating roles of initiated and received task interdependence as proxies for team coupling structure. A field survey study of 110 employees, who worked in distributed teams in three Norwegian organizations, showed that when there are high levels of initiated task interdependence and low levels of received task interdependence, team self- management is associated with stronger perceived team coordination in distributed teams. Theoretical and practical implications for distributed self- managing teams are also discussed.

\section{Paper 3: Coordination in OSS 2.0: ANT Approach}

Paper number three is our Best Paper nominee. It focuses on the perennial problem of coordination in teams, but in the case in this paper focuses on Open Source Software (OSS) teams. Open source software projects are increasingly driven by a combination of independent and professional developers, the former volunteers and the later hired by a company to contribute to the project to support commercial product development. This mix of developers has been referred to as OSS 2.0. However, the authors argued that they did not fully understand the multi-layered coordination spanning individuals, teams, and organizations. Using Actor-Network Theory (ANT), they described how coordination and power dynamics unfold among developers and how different tools and artifacts both display activities and mediate coordination efforts. Internal communication within an organization was reported to cause broken links in the community, duplication of work, and political tensions. ANT shows how tools and code can exercise agency and alter a software development process as an equivalently active actor of the scene. They end with a discussion of the theoretical and practical implications of the changing nature of open source software development.

\section{Paper 4: Using Social Relations Analysis to Examine the Impact of Role Change on Interpersonal Perceptions in Virtual Teams}

The final paper demonstrates a statistical strategy for examining how role disruptions might affect interpersonal perceptions in virtual teams. Specifically, the authors describe how to employ a social relations analysis to uncover interpersonal processes within virtual teams that might be hidden in the complex multilevel structure of teams. The demonstration relies on data from a study involving 40 virtual teams configured in a laboratory; half of the teams experienced a change in leader role during the collaboration. The analysis revealed systematic rater and relationship patterns in how team members evaluated each other. Teams experiencing role change showed more differentiation of partners in evaluations and accuracy in guessing how team members evaluated them, compared to teams without role changes.

\section{Towards a HICSS VTONs Community}

While VTON's has grown in popularity, to the point that practitioners often no longer distinguish them from more permanent teams, there are still so many challenges that remain unresolved in research. This led to our decision to expand this mini-track a few years ago. This expansion has demonstrated great potential to stimulate the creation of a robust, interdisciplinary HICSS community studying virtual teams, organizations, and networks (VTONs). Given the increasing use of VTONs in industry, academia, medicine, and civil society, such a community would be invaluable. The VTONs papers at this $52^{\text {nd }}$ HICSS represent what we see as an important trend, which we believe will remain for many years to come. It is a privilege to bring you these exciting papers and we look forward to having productive and stimulating discussions about current and future VTONs issues. 